\title{
Versorgungssicherheit und Engpassmanagement
}

Die großflächigen Versorgungsausfälle im Jahr 2003 haben die Aufmerksamkeit der Öffentlichkeit auf die Energiesysteme gelenkt. Derzeit haben wir weiterhin einen jährlichen Zuwachs des Elektrizitätsbedarfs von etwa $2 \%$. Die Energiesysteme können aber nicht entsprechend diesem Zuwachs ausgebaut werden. Die Ursachen hierfür sind vielfältig. Sie reichen von langen Verzögerungen bei der Genehmigung von dringend benötigten Teilabschnitten des 380-kV-Übertragungsnetzes über erhöhte Netzbelastungen durch den weiträumigeren Strommarkt bis zur Abschaltung von unwirtschaftlichen alten Kraftwerken und langen Vorlaufzeiten für den Neubau dieser Einheiten.

Viele thermische Kraftwerke erreichen im Zeitraum von 2005 bis 2015 ihre technische Lebensdauergrenze. In Österreich besteht dabei ein Bedarf an Neubau von Kraftwerken in der Größenordnung von $3000 \mathrm{MW}$ bis $5000 \mathrm{MW}$. Innerhalb der EU-15-Länder wird bis 2030 mit einem Bedarf von $600000 \mathrm{MW}$ gerechnet.

Die Erzeugungslücke kann wegen der begrenzten Ressourcen und des hohen Förderungsbedarfs nur teilweise durch regenerative Energie abgedeckt werden. Der überwiegende Anteil wird aus thermischen Kraftwerken bereitgestellt werden müssen, insbesondere aus hoch effizienten Gas- und Dampfkraftwerken mit geringen Emissionen. Ein weiterer Teil ist zukünttig durch Importe zu decken.

Der Neubau der Kraftwerke hat unter den veränderlichen Bedingungen des freien Strommarktes, der Emissionszertifizierung bzw. dem Allokationsplan für $\mathrm{CO}_{2}-E$ missionen, die zum Zeitpunkt der Errichtung gelten, zu erfolgen. Die langen Planungszeiträume und Genehmigungsverfahren für Kraftwerke und Leitungen machen ein baldiges Handeln und daher beschleunigte Genehmigungen erforderlich.

Die unzureichende Übertragungskapazität in Österreich erschwert derzeit die Entwicklung des freien Strommarktes. Die

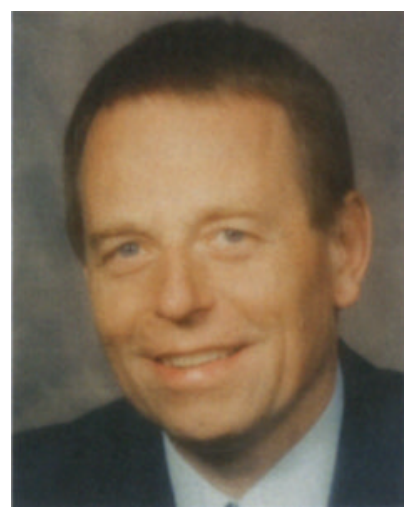

Günther Brauner

erforderlichen Maßnahmen für das Engpassmanagement verteuern den Strom. Zur Planung der erforderlichen Maßnahmen und zur Minderung des Risikos von Großstörungen sind Simulationsstudien erforderlich, mit denen die Wirksamkeit und Wirtschaftlichkeit verschiedener Optionen verglichen werden können. Des Weiteren können hiermit die Auswirkungen der neuen Rahmenbedingungen wie dem Gesetz zur Emissionsminderung und der Wasserrahmenrichtlinien auf die Erzeugungskapazitäten und den Neubau von Kraftwerken bzw. die Schaffung von Leitungskapazitäten für den Stromimport untersucht werden.

Mit dieser e \& i-Ausgabe wollen wir auf diese Problematiken aufmerksam machen und Lösungswege aufzeigen.

\section{O. Univ.-Prof. Dipl.-Ing. Dr.-Ing. Günther Brauner OVE}

Institut für Elektrische Anlagen und Energiewirtschaft Technische Universität Wien 